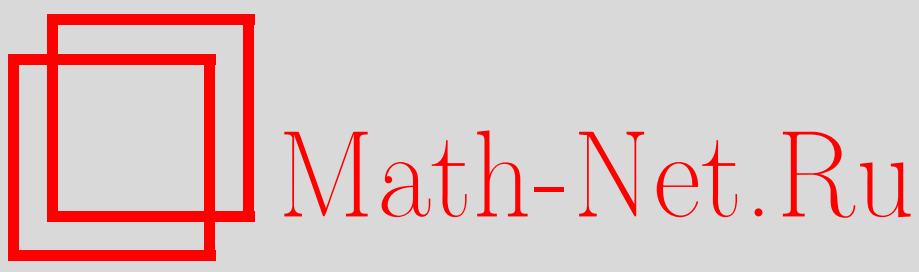

Г. Г. Лаптев, Отсутствие решений полулинейных параболических дифференциальных неравенств в конусах, Матем. сб., 2001, том 192, номер 10, 51-70

DOI: https://doi.org/10.4213/sm602

Использование Общероссийского математического портала Math-Net.Ru подразумевает, что вы прочитали и согласны с пользовательским соглашением

http://www . mathnet.ru/rus/agreement

Параметры загрузки:

IP: 54.197 .130 .99

26 апреля 2023 г., 16:07:34 
УДК 517.9

\author{
Г.Г. Лаптев
}

\title{
Отсутствие решений полулинейных параболических дифференциальньх неравенств в конусах
}

\begin{abstract}
Исследуются вопросы отсутствия глобальных нетривиальных решений полулинейных параболических дифференциальных неравенств и систем второго порядка в конусах. Доказательство основано на методе пробных функций без использования теорем сравнения и принципа максимума.

Библиография: 22 названия.
\end{abstract}

\section{$\S 1$. Введение}

Работа посвящена нахождению условий отсутствия нетривиальных глобальных решений полулинейных дифференциальных неравенств параболического типа в конических областях. Постановка задачи предполагает неограниченность области и существование неотрицательного локального решения задачи. Проблема возникает при попытке продолжить решение до глобального, т.е. найти решение задачи Коши, определенное на всей рассматриваемой области. При этом даже для полулинейных задач вида

$$
\begin{aligned}
& \frac{\partial u}{\partial t}-\Delta u=u^{q} \quad \text { в } \mathbb{R}^{N} \times(0, \infty), \\
& u(x, 0)=u_{0}(x) \geqslant 0 \quad \text { в } \mathbb{R}^{N}
\end{aligned}
$$

существует так называемый критический показатель нелинейности (в данном случае показатель Фужиты $\left.q^{*}=1+2 / N\right)$ такой, что при $1<q \leqslant q^{*}$ любое локальное по переменной $t$ решение нельзя продолжить до глобального (под словом “продолжить" понимается, естественно, продолжение с сохранением принадлежности некоторому локальному функциональному пространству). Такого рода утверждения формулируются также в виде теорем об отсутствии глобального решения. Удивительным фактом здесь является то, что при этом не делается каких-либо предположений о росте глобального решения на бесконечности. Более детальная постановка задачи и обзор литературы имеются в [1].

Вместо приведенного выше уравнения можно рассматривать дифференциальное неравенство в локальном пространстве дважды непрерывно дифференцируемых

Работа вьполнена при финансовой поддержке INTAS (грант №971-30551), Программы поддержки ведущих научных школ (грант № 00-15-96047) и Российского фонда фундаментальных исследований (грант № 99-01-00045). 
функций без начальных условий

$$
\begin{gathered}
\frac{\partial u}{\partial t}-\Delta u \geqslant u^{q} \quad \text { в } \mathbb{R}^{N} \times(0, \infty), \\
u(x, t) \geqslant 0 \quad \text { в } \mathbb{R}^{N} \times(0, \infty), \\
u \neq 0 .
\end{gathered}
$$

При этом также возникает критический показатель, совпадающий с аналогичным показателем для уравнения (что часто не имеет места для эллиптических и гиперболических неравенств). Интересной особенностью неравенств является возможность в некоторых случаях явно построить решения для суперкритической нелинейности $\left(q>q^{*}\right)$ и таким образом легко установить неулучшаемость полученных результатов.

В настояшей статье рассматривается выписанное выше неравенство (и различные обобщения) в случае, когда вместо всего пространства $\mathbb{R}^{N}$ берется конус $K$. При этом критический показатель зависит от характеристики конуса, которая выражается через первое собственное значение соответствующей эллиптической задачи на единичной сфере.

Теория линейных краевых задач в конических областях берет свое начало с фундаментальной работы В.А. Кондратьева [2] по эллиптическим уравнениям. Вопросы отсутствия решений соответствующих полулинейных и нелинейных эллиптических и параболических задач изучаются, главным образом, путем сведения к интегральному уравнению и с использованием утверждений типа теорем сравнения и принципа максимума. Для случая параболических уравнений отметим работы [3], [4] и ставшую уже классической книгу [5]. Современное состояние исследований отражено в обзорах [6], [7].

Для эллиптических уравнений и неравенств основанные на теоремах сравнения результаты для различных областей см. в [8]-[11]. Следствием этих оценок является, в частности, отсутствие нетривиального решения при некоторых дополнительных условиях.

В настоящей работе для доказательства отсутствия решения применяется метод пробных функций [12]-[18] без использования характерных для теории уравнений второго порядка принципов сравнения. Этот метод дает возможность с использованием техники Э. Митидиери и С.И. Похожаева [14] практически сразу получать отсутствие решения для критического показателя нелинейности (что раньше вызывало существенные затруднения), а также рассматривать системы дифференциальных неравенств и другие классы задач, для которых принцип максимума не имеет места. Основной задачей становится выбор пробной функции и оценка соответствующих интегралов от нее. Отсутствие решений эллиптических неравенств в неограниченных конических областях методом пробных функций изучалось в работе автора [19].

Пусть $K_{\omega}$ - область на единичной сфере $S^{N-1} \subset \mathbb{R}^{N}, N \geqslant 3$, с кусочно гладкой границей $\partial K_{\omega}$. Конусом $K$ назьвается множество, которое в сферической системе координат $(r, \omega), 0 \leqslant r<\infty, \omega \in S^{N-1}$, имеет вид

$$
K=\left\{x=(r, \omega): 0<r<+\infty, \omega \in K_{\omega}\right\}
$$

Боковую поверхность конуса обозначаем через $\partial K$. 
Пусть $\Omega$ - неограниченная область пространства $\mathbb{R}^{N+1}$ с кусочно гладкой границей. Далее используются анизотропные пространства С.Л. Соболева $W_{q}^{2,1}(\Omega)$, а также локальное пространство $L_{q, \text { loc }}(\Omega)$, элементы которого принадлежат $L_{q}\left(\Omega^{\prime}\right)$ для любого компактного подмножества $\Omega^{\prime}: \bar{\Omega}^{\prime} \subset \Omega$. Через $C(\bar{\Omega})$ обозначается пространство непрерывных функций, через $C^{m}(\bar{\Omega})-$ пространство гладких функций на замкнутой области $\bar{\Omega}$. Символ $\Delta$ обозначает оператор Лапласа, вектор из частных производных обозначаем через $D u=\left(\frac{\partial u}{\partial x_{i}}\right)$. Для двух дифференцируемых функций $u(x)$ и $\varphi(x)$ полагаем $D u D \varphi=\sum_{i=1}^{N} \frac{\partial u}{\partial x_{i}} \frac{\partial \varphi}{\partial x_{i}}$. Выражение $\frac{\partial u}{\partial n}$ обозначает производную функции $u$ по направлению внешней нормали $n$ к границе конуса $\partial K$. Через $c$ и $C$ с индексами будем обозначать постоянные.

Напомним, что оператор Лапласа $\Delta$ в сферических координатах $(r, \omega)$ имеет вид

$$
\Delta=\frac{1}{r^{N-1}} \frac{\partial}{\partial r}\left(r^{N-1} \frac{\partial}{\partial r}\right)+\frac{1}{r^{2}} \Delta_{\omega}=\frac{\partial^{2}}{\partial r^{2}}+\frac{N-1}{r} \frac{\partial}{\partial r}+\frac{1}{r^{2}} \Delta_{\omega}
$$

г де $\Delta_{\omega}$ - оператор Бельтрами-Лапласа на единичной сфере $S^{N-1} \subset \mathbb{R}^{N}$.

В дальнейшем постоянно используются наименьшее (первое) собственное значение $\lambda_{\omega} \equiv \lambda_{1}\left(K_{\omega}\right)>0$ и соответствующая собственная функция $\Phi(\omega)$ оператора $\Delta_{\omega}$, являюшиеся решением задачи

$$
\begin{aligned}
& \Delta_{\omega} \Phi+\lambda \Phi=0 \text { в } K_{\omega}, \\
& \left.\Phi\right|_{\partial K_{\omega}}=0 .
\end{aligned}
$$

Хорошо известно, что $\Phi(\omega)>0$ для $\omega \in K_{\omega}$. Предполагается, что $\Phi(\omega) \leqslant 1$.

\section{§2. Оценки некоторых интегралов емкостного типа}

Как было упомянуто во введении, главным моментом в используемом методе является подходящий выбор пробных функций и оценка интегралов специального вида. В настоящем параграфе мы проводим соответствующие построения, которые применяются во всех последующих утверждениях.

Пробные функции мы строим методом разделения переменных, поэтому сначала рассмотрим соответствуюшую функцию по переменной $t$, а затем по $x$.

Напомним хорошо известный факт существования бесконечно дифференцируемой функции $\zeta(y) \in C^{\infty}\left(\mathbb{R}_{+}\right)$(так называемой “стандартной срезающей функции") со следующими свойствами:

$$
0 \leqslant \zeta(y) \leqslant 1, \quad \zeta(y)=\left\{\begin{array}{l}
1, \text { если } 0 \leqslant y \leqslant 1 \\
0, \text { если } y \geqslant 2
\end{array}\right.
$$

Такого рода функции широко используются в теории функций в процедурах усреднения [20]. 
Следующим шагом является построение функции $\eta(y)$ такой, чтобы при фиксированном $p_{0}$ для всех $1<p \leqslant p_{0}$ были выполнены поточечные неравенства

$$
\left|\eta^{\prime}(y)\right|^{p} \leqslant c_{\eta} \eta^{p-1}(y), \quad\left|\eta^{\prime \prime}(y)\right|^{p} \leqslant c_{\eta} \eta^{p-1}(y)
$$

с некоторой постоянной $c_{\eta}$. Достаточно взять

$$
\eta(y)=(\zeta(y))^{2 p_{0}}
$$

Тогда

$$
\begin{gathered}
\eta^{\prime}(y)=2 p_{0} \zeta^{2 p_{0}-1} \zeta^{\prime}, \quad\left|\eta^{\prime}(y)\right|^{p}=\left(2 p_{0}\right)^{p} \zeta^{2 p_{0}(p-1)} \zeta^{2 p_{0}-p}\left|\zeta^{\prime}\right|^{p} \leqslant c_{\eta} \eta^{p-1}(y) \\
\eta^{\prime \prime}(y)=2 p_{0}\left(\left(2 p_{0}-1\right) \zeta^{2 p_{0}-2}\left|\zeta^{\prime}\right|^{2}+\zeta^{2 p_{0}-1} \zeta^{\prime \prime}\right) \\
\left|\eta^{\prime \prime}(y)\right|^{p} \leqslant\left(2 p_{0}\right)^{p} \zeta^{2 p_{0}(p-1)} \zeta^{2 p_{0}-2 p}\left(\left(2 p_{0}-1\right)\left|\zeta^{\prime}\right|^{2}+\zeta\left|\zeta^{\prime \prime}\right|\right)^{p} \leqslant c_{\eta} \eta^{p-1}(y) .
\end{gathered}
$$

Введем параметр $\rho$, который в последующих построениях будет неограниченно возрастать, и положительную степень $\theta$. Введем также функцию $\eta\left(t / \rho^{\theta}\right)$ переменной $t \geqslant 0$. Для используемого в дальнейшем интеграла с помощью замены переменных получаем оценку

$$
\int_{\operatorname{supp}\left|\frac{d \eta\left(t / \rho^{\theta}\right)}{d t}\right|} \mid \frac{\left.\frac{d \eta\left(t / \rho^{\theta}\right)}{d t}\right|^{p}}{\eta^{p-1}\left(t / \rho^{\theta}\right)} d t \leqslant c_{\eta} \rho^{-\theta(p-1)}
$$

При этом

$$
\operatorname{supp}\left|\eta\left(\frac{t}{\rho^{\theta}}\right)\right|=\left\{t<2 \rho^{\theta}\right\}, \quad \operatorname{supp}\left|\frac{d \eta\left(t / \rho^{\theta}\right)}{d t}\right|=\left\{\rho^{\theta}<t<2 \rho^{\theta}\right\} .
$$

Аналогичные построения проведем и для пробной функции по переменной $x$. Однако в данном случае в силу того, что мы рассматриваем коническую область, возникает произведение двух функций:

$$
r^{s} \eta\left(\frac{r}{\rho}\right)
$$

с некоторым положительным $s$. Отсюда

$$
\frac{\partial\left(r^{s} \eta(r / \rho)\right)}{\partial r}=s r^{s-1} \eta\left(\frac{r}{\rho}\right)+r^{s} \eta^{\prime}\left(\frac{r}{\rho}\right) \frac{1}{\rho}
$$

и

$$
\begin{aligned}
\left|\frac{\partial\left(r^{s} \eta(r / \rho)\right)}{\partial r}\right|^{p} & \leqslant c_{p} s^{p} r^{p(s-1)} \eta^{p}\left(\frac{r}{\rho}\right)+c_{p} r^{p s}\left|\eta^{\prime}\left(\frac{r}{\rho}\right)\right|^{p} \frac{1}{\rho^{p}} \\
& \leqslant c_{p s \eta} \eta^{p-1}\left(\frac{r}{\rho}\right) r^{p(s-1)}\left(1+\frac{r^{p}}{\rho^{p}}\right)
\end{aligned}
$$


с не зависящей от $\rho$ и $r$ постоянной $c_{p s \eta}$. Такого же рода оценку можно установить и для второй производной:

$$
\frac{\partial^{2}\left(r^{s} \eta(r / \rho)\right)}{\partial r^{2}}=r^{s-2}\left(s(s-1) \eta\left(\frac{r}{\rho}\right)+2 s \eta^{\prime}\left(\frac{r}{\rho}\right) \frac{r}{\rho}+\eta^{\prime \prime}\left(\frac{r}{\rho}\right) \frac{r^{2}}{\rho^{2}}\right)
$$

и

$$
\left|\frac{\partial^{2}\left(r^{s} \eta(r / \rho)\right)}{\partial r^{2}}\right|^{p} \leqslant c_{p s \eta} \eta^{p-1}\left(\frac{r}{\rho}\right) r^{p(s-2)}\left(1+\frac{r^{p}}{\rho^{p}}+\frac{r^{2 p}}{\rho^{2 p}}\right) .
$$

Теперь проясним смысл проведенных оценок. Для этого обратимся уже непосредственно к рассматриваемому нами конусу и оператору Лапласа $\Delta$. Введем функцию $r^{s} \Phi(\omega)$. Вычисляя $\Delta\left(r^{s} \Phi(\omega)\right)$, с учетом равенства $\Delta_{\omega} \Phi=-\lambda_{\omega} \Phi$ приходим к выражению

$$
\Delta\left(r^{s} \Phi(\omega)\right)=r^{s-2} \Phi(\omega)\left\{s(s-1)+s(N-1)-\lambda_{\omega}\right\}
$$

Введем параметры

$$
s^{*}=\frac{N-2}{2}+\sqrt{\left(\frac{N-2}{2}\right)^{2}+\lambda_{\omega}}, \quad s_{*}=-\frac{N-2}{2}+\sqrt{\left(\frac{N-2}{2}\right)^{2}+\lambda_{\omega}} .
$$

Числа $s_{*}$ и $-s^{*}$ являются корнями многочлена в фигурных скобках в предыдущем выражении для $\Delta\left(r^{s} \Phi(\omega)\right)$. Заметим, что $s^{*}-s_{*}=N-2$. Введем функцию

$$
\xi(x) \equiv \xi(r, \omega)=r^{s *} \Phi(\omega) .
$$

Главньм ее свойством является тождество $\Delta \xi=0$ в $K$. Очевидно также, что она обращается в нуль на $\partial K$. Еще одним используемым далее свойством функции (2.4) является неположительность производной $\frac{\partial \xi}{\partial n}$ по внешней нормали к границе конуса $\partial K$. Действительно,

$$
\frac{\partial \xi}{\partial n}=r^{s_{*}} \frac{\partial \Phi(\omega)}{\partial n_{\omega}} \leqslant 0
$$

где $n_{\omega}$ - внешняя нормаль к границе области $K_{\omega}$, а неположительность производной $\frac{\partial \Phi(\omega)}{\partial n_{\omega}}$ следует из леммы Хопфа (так как сама функция $\Phi(\omega)$ положительна в $K_{\omega}$ и обращается в нуль на гранище).

Теперь установим аналогичную (2.1) оценку для “срезанной” функции

$$
\psi_{\rho}(x)=\xi(x) \eta\left(\frac{|x|}{\rho}\right)=r^{s_{*}} \eta\left(\frac{r}{\rho}\right) \Phi(\omega) .
$$

Для этого сначала заметим, что в сферических координатах получаем:

$$
\Delta \psi_{\rho}=\frac{\partial^{2} \psi_{\rho}}{\partial r^{2}}+\frac{N-1}{r} \frac{\partial \psi_{\rho}}{\partial r}+\frac{1}{r^{2}} \Delta_{\omega} \psi_{\rho}
$$

где

$$
\Delta_{\omega} \psi_{\rho}=r^{s_{*}} \eta\left(\frac{|x|}{\rho}\right)\left(-\lambda_{\omega} \Phi\right)=-\lambda_{\omega} \psi_{\rho} .
$$


С учетом установленных выше оценок для производных от произведения $r^{s} \eta(r / \rho)$ будем иметь

$$
\begin{aligned}
\left|\Delta \psi_{\rho}(x)\right|^{p} & =\left|\left(\frac{\partial^{2}}{\partial r^{2}}+\frac{N-1}{r} \frac{\partial}{\partial r}-\frac{\lambda_{\omega}}{r^{2}}\right)\left(r_{*}^{s_{*}} \eta\left(\frac{r}{\rho}\right)\right)\right|^{p} \Phi^{p}(\omega) \\
& \leqslant c \eta^{p-1}\left(\frac{r}{\rho}\right) \Phi^{p}(\omega) \frac{r^{(p-1) s_{*}}}{r^{2 p-s_{*}}}\left(1+\frac{r^{p}}{\rho^{p}}+\frac{r^{2 p}}{\rho^{2 p}}\right) \\
& \leqslant c \psi_{\rho}^{p-1}(x) \frac{1}{r^{2 p-s_{*}}}\left(1+\frac{r^{p}}{\rho^{p}}+\frac{r^{2 p}}{\rho^{2 p}}\right)
\end{aligned}
$$

По построению $\eta(|x| / \rho) \equiv 1$ при $|x| \leqslant \rho$, поэтому $\Delta \psi_{\rho}(x)=\Delta \xi(x)=0$ и $\operatorname{supp}\left|\Delta \psi_{\rho}(x)\right|=\{K \cap\{\rho<|x|<2 \rho\}\}$. Отсюда следует, что на множестве $\operatorname{supp}\left|\Delta \psi_{\rho}(x)\right|$ в приведенной выше оценке выражение в круглых скобках ограничено. Далее, на этом множестве $r^{s^{*}-2 p} \leqslant C \rho^{s^{*}-2 p}$ (независимо от знака $s^{*}-2 p$ ), поэтому

$$
\left|\Delta \psi_{\rho}(x)\right|^{p} \leqslant c \psi_{\rho}^{p-1}(x) \rho^{s_{*}-2 p}
$$

Теперь можем окончательно записать

$$
\int_{\operatorname{supp}\left|\Delta \psi_{\rho}\right|} \frac{\left|\Delta \psi_{\rho}(x)\right|^{p}}{\psi_{\rho}^{p-1}(x)} d x \leqslant c \int_{\rho}^{2 \rho} \int_{K_{\omega}} \frac{\psi_{\rho}^{p-1}(x)}{\psi_{\rho}^{p-1}(x)} \frac{r^{N-1}}{\rho^{2 p-s_{*}}} d r d \omega \leqslant c_{\psi} \rho^{-2 p+s_{*}+N}
$$

Общую пробную функцию выбираем в виде произведения

$$
\varphi_{\rho}(x, t)=\eta\left(\frac{t}{\rho^{\theta}}\right) \psi_{\rho}(x) .
$$

С учетом изложенного вьшше очевидны оценки

$$
\begin{aligned}
& \iint_{\operatorname{supp}\left|\frac{\partial \varphi_{\rho}}{\partial t}\right| \frac{\left|\frac{\partial \varphi_{\rho}(x, t)}{\partial t}\right|^{p}}{\varphi_{\rho}^{p-1}(x, t)} d x d t} \leqslant \int_{K \cap\{r<2 \rho\}} \psi_{\rho}(x) d x \int_{\operatorname{supp}\left|\frac{d \eta\left(t / \rho^{\theta}\right)}{d t}\right| \frac{\left|\frac{d \eta\left(t / \rho^{\theta}\right)}{d t}\right|^{p}}{\eta^{p-1}\left(t / \rho^{\theta}\right)} d t} \\
& \leqslant c_{\varphi} \rho^{s_{*}+N-\theta(p-1)}, \\
& \iint_{\operatorname{supp}\left|\Delta \varphi_{\rho}\right|} \frac{\left|\Delta \varphi_{\rho}(x, t)\right|^{p}}{\varphi_{\rho}^{p-1}(x, t)} d x d t \leqslant \int_{0}^{2 \rho^{\theta}} \eta\left(\frac{t}{\rho^{\theta}}\right) d t \int_{\operatorname{supp}\left|\Delta \psi_{\rho}\right|} \frac{\left|\Delta \psi_{\rho}\right|^{p}}{\psi_{\rho}^{p-1}} d x \\
& \leqslant c_{\varphi} \rho^{\theta-2 p+s_{*}+N} .
\end{aligned}
$$

При $\theta=2$ степени в правых частях неравенств (2.7) уравниваются, поэтому окончательно получаем оценку

$$
\iint_{\operatorname{supp}\left|\frac{\partial \varphi_{\rho}}{\partial t}+\Delta \varphi_{\rho}\right|} \frac{\left|\frac{\partial \varphi_{\rho}}{\partial t}+\Delta \varphi_{\rho}\right|^{p}}{\varphi_{\rho}^{p-1}} d x d t \leqslant c_{0} \rho^{-2 p+s_{*}+N+2} .
$$

ЗАмЕчАнИЕ 1. Приведенные построения можно провести различными способами. Основная идея состоит в “срезании” функций, на которых рассматриваемый 
оператор обрашается в нуль, таким образом, чтобы они становились финитньми и в дальнейшем при интегрировании по частям не порождали граничных интегралов.

В простейшем случае одной производной, который мы и имеем по переменной $t$, в качестве такой срезаемой функции берется тождественная единица. Для оператора Лапласа (в данном контексте) мы вынуждены использовать уже более сложные функции типа фундаментальных решений.

Само построение в виде умножения на стандартную срезающую функцию придает некоторую наглядность дальнейшим оценкам, однако можно и явно построить требуемую функцию в виде степеней некоторого многочлена второго порядка и оценить все получаемые интегралы, как это проделано в работе автора [19].

ЗАмЕчАниЕ 2. Такого рода оценки для случая всего пространства $\mathbb{R}^{N}$ применяются в работах [13]-[16] и по сушеству являются строгим подходом к широко используемой для предварительного определения критических показателей технике замены переменных ("scaling") [7].

ЗАмечАниЕ 3. Параметр $\theta$, который в данном построении взят равньм 2 , в дальнейшем будет варьироваться в зависимости от главного оператора. Мы будет делать соответствующие замечания по ходу изложения, не останавливаясь детально на построении соответствующих функций и оценках интегралов вида (2.8).

\section{§ 3. Модельная задача: отсутствие глобального решения}

Рассмотрим проблему отсутствия слабых решений задачи

$$
\begin{gathered}
\frac{\partial u}{\partial t}-\Delta u \geqslant u^{q} \quad \text { в } K \times(0, \infty), \\
u \geqslant 0, \quad u \neq 0 .
\end{gathered}
$$

Далее слабое решение будет пониматься в следуюшем смысле.

ОПРЕДЕЛЕНИЕ 1. Пусть $u(x, t) \in C(\bar{K} \times[0, \infty))$. Неотрицательная функция $u(x, t)$ называется слабым решением задачи (3.1), если для любой неотрицательной пробной функции $\varphi(x, t) \in W_{\infty}^{2,1}(K \times(0, \infty))$ такой, что $\left.\varphi\right|_{\partial K \times(0, \infty)}=0$, финитной по переменным $r=|x|$ и $t$, выполнено интегральное неравенство

$$
\begin{gathered}
\int_{0}^{\infty} \int_{\partial K} u \frac{\partial \varphi}{\partial n} d x d t-\int_{0}^{\infty} \int_{K} u\left(\frac{\partial \varphi}{\partial t}+\Delta \varphi\right) d x d t \\
\geqslant \int_{0}^{\infty} \int_{K} u^{q} \varphi d x d t+\int_{K} u(x, 0) \varphi(x, 0) d x
\end{gathered}
$$

ЗАмЕчАниЕ 4. Приведенное определение обобшенного решения может быть расширено, в частности, рассмотрением пространства локально суммируемых функций $L_{q, \text { loc }}(K)$ вместо пространства непрерывных функций. При этом нужно делать дополнительные предположения о следе функции $u(x, t)$ на границе конуса и при $t=0$, однако в обшем случае это не отразится на получаемом критическом показателе. 
TEOPEMA 1. $\Pi p u$

$$
1<q \leqslant q^{*}=1+\frac{2}{s^{*}+2}
$$

задача (3.1) не имеет нетривиального глобального решения.

ЗАмечАниЕ 5. Параметры $s_{*}$ и $s^{*}$ возникают при построении фундаментальных решений эллиптического оператора $\Delta$ в конусе $K$. Как будет видно ниже, фундаментальных решений эллиптического оператора достаточно для получения неулучшаемого критического показателя $q^{*}$, т.е. нет необходимости переходить к существенно более сложному фундаментальному решению оператора теплопроводности $\partial / \partial t-\Delta($ см. также [18]).

ДокАЗАТЕЛЬСтво теоремЫ 1. От противного. Пусть $u(x, t)$ - нетривиальное решение задачи (3.1) с $1<q \leqslant q^{*}$. Согласно определению 1 с пробной функцией $\varphi(x, t)=\varphi_{\rho}(x, t)$, введенной по формуле $(2.6)$ с $p=q^{\prime}>1$ и $\theta=2$, это означает

$$
\begin{aligned}
& \int_{K} u(x, 0) \varphi_{\rho}(x, 0) d x+\int_{0}^{\infty} \int_{K} u^{q} \varphi_{\rho} d x d t \\
& \quad \leqslant \int_{0}^{\infty} \int_{\partial K} u \frac{\partial \varphi_{\rho}}{\partial n} d x d t-\iint_{\operatorname{supp}\left|\frac{\partial \varphi_{\rho}}{\partial t}+\Delta \varphi_{\rho}\right|} u\left(\frac{\partial \varphi_{\rho}}{\partial t}+\Delta \varphi_{\rho}\right) d x d t
\end{aligned}
$$

Как уже отмечалось ранее, производная функции $\xi$, а следовательно, и $\varphi_{\rho}$ по нормали к границе конуса будет неположительна, и поскольку функции $u$ и $\frac{\partial \varphi \rho}{\partial n}$ непрерывны на границе $\partial K \times[0, \infty)$, включая вершину $x=0$, то первый интеграл в правой части (по границе) не будет иметь особенностей и будет неположительным.

Для оценки второго интеграла применим неравенство Гёльдера. Получим:

$$
\begin{aligned}
\int_{K} u & (x, 0) \varphi_{\rho}(x, 0) d x+\int_{0}^{\infty} \int_{K} u^{q} \varphi_{\rho} d x d t \\
= & \int_{K} u(x, 0) \varphi_{\rho}(x, 0) d x+\iint_{\operatorname{supp}\left|\frac{\partial \varphi_{\rho}}{\partial t}+\Delta \varphi_{\rho}\right|} u^{q} \varphi_{\rho} d x d t \\
& +\iint_{\varphi_{\rho}(x, t)=\xi(x)} u^{q} \xi(x) d x d t \\
\leqslant & \iint_{\operatorname{supp}\left|\frac{\partial \varphi_{\rho}}{\partial t}+\Delta \varphi_{\rho}\right|} u\left|\frac{\partial \varphi_{\rho}}{\partial t}+\Delta \varphi_{\rho}\right| d x d t \\
\leqslant & \left(\iint_{\operatorname{supp}\left|\frac{\partial \varphi_{\rho}}{\partial t}+\Delta \varphi_{\rho}\right|} u^{q} \varphi_{\rho} d x d t\right)^{1 / q} \\
& \times\left(\iint_{\operatorname{supp}\left|\frac{\partial \varphi_{\rho}}{\partial t}+\Delta \varphi_{\rho}\right|} \frac{\left|\frac{\partial \varphi_{\rho}}{\partial t}+\Delta \varphi_{\rho}\right|^{q^{\prime}}}{\varphi_{\rho}^{q^{\prime}-1}} d x d t\right)^{1 / q^{\prime}}
\end{aligned}
$$

откуда, отбрасьвая неотрицательные начальные условия (т.е. первый интеграл в левой части), с учетом оценки (2.8) (при $p=q^{\prime}$ ) для последнего интеграла справа 
будем иметь

$$
\begin{aligned}
\iint_{\varphi_{\rho}(x, t)=\xi(x)} u^{q} \xi(x) d x d t & \leqslant \iint_{\operatorname{supp}\left|\frac{\partial \varphi_{\rho}}{\partial t}+\Delta \varphi_{\rho}\right|} \frac{\left|\frac{\partial \varphi_{\rho}}{\partial t}+\Delta \varphi_{\rho}\right|^{q^{\prime}}}{\varphi^{q^{\prime}-1}} d x d t \\
& \leqslant c_{0} \rho^{-2 q^{\prime}+s_{*}+N+2} .
\end{aligned}
$$

Так как подынтегральное выражение в левой части не зависит от $\rho$, можем перейти к пределу по $\rho \rightarrow \infty$. В случае

$$
-2 q^{\prime}+s_{*}+N+2 \leqslant 0
$$

это приводит к соотношению

$$
\int_{0}^{\infty} \int_{K} u^{q} \xi d x d t \leqslant c_{0}
$$

Тогда в силу абсолютной непрерывности интеграла Лебега по мере и ввиду оценки $\varphi_{\rho} \leqslant \xi$

$$
\iint_{\operatorname{supp}\left|\frac{\partial \varphi_{\rho}}{\partial t}+\Delta \varphi_{\rho}\right|} u^{q} \varphi_{\rho} d x d t \leqslant \iint_{\operatorname{supp}\left|\frac{\partial \varphi_{\rho}}{\partial t}+\Delta \varphi_{\rho}\right|} u^{q} \xi d x d t=\varepsilon(\rho) \rightarrow 0
$$

при $\rho \rightarrow \infty$.

Возвращаясь теперь к неравенству (3.3), получаем

$$
\iint_{\varphi_{\rho}(x, t)=\xi(x)} u^{q} \xi d x d t \leqslant \varepsilon^{1 / q}(\rho) c_{0}^{1 / q^{\prime}} \rightarrow 0
$$

при $\rho \rightarrow \infty$, т.е. в пределе

$$
\int_{0}^{\infty} \int_{K} u^{q} \xi d x d t=0
$$

откуда в силу положительности $\xi$ в $K$ следует $u \equiv 0$, что противоречит нашему предположению о сушествовании нетривиального решения ( техника исследования критического случая $-2 q^{\prime}+s_{*}+N+2=0$ заимствована из работы [14]).

Элементарньми преобразованиями из неравенства

$$
-2 q^{\prime}+s_{*}+N+2 \leqslant 0
$$

получаем условие отсутствия нетривиального решения: $1<q \leqslant q^{*}$.

ЗАмЕчАнИЕ 6. Полученный критический показатель является неулучшаемьм, т.е. в случае $q>q^{*}$ существуют глобальные нетривиальные положительные решения рассматриваемой задачи и даже соответствуюшего уравнения. 


\section{§4. Задачи с переменными коэффициентами}

В настояшем параграфе приведем пример применения описанного метода к исследованию отсутствия решения параболических задач с переменными коэффициентами.

Рассмотрим проблему отсутствия слабых решений задачи

$$
\begin{gathered}
\frac{\partial u}{\partial t}-\operatorname{div}\left(|x|^{\alpha} D u\right) \geqslant u^{q} \text { в } K \times(0, \infty), \\
u \geqslant 0, \quad u \neq \equiv .
\end{gathered}
$$

Здесь $2>\alpha>1-N$. Далее слабое решение будет пониматься в следуюшем смысле.

ОПРЕДЕЛЕНИЕ 2. Пусть $u(x, t) \in C(\bar{K} \times[0, \infty))$. Неотрицательная функция $u(x, t)$ называется слабым решением задачи (4.1), если для любой неотрицательной пробной функции $\varphi(x, t) \in W_{\infty}^{2,1}(K \times(0, \infty))$ такой, что $\left.\varphi\right|_{\partial K \times(0, \infty)}=0$, финитной по переменным $r=|x|$ и $t$, выполнено интегральное неравенство

$$
\begin{aligned}
\int_{0}^{\infty} & \int_{\partial K} u|x|^{\alpha} \frac{\partial \varphi}{\partial n} d x d t-\int_{0}^{\infty} \int_{K} u\left(\frac{\partial \varphi}{\partial t}+\operatorname{div}\left(|x|^{\alpha} D \varphi\right)\right) d x d t \\
& \geqslant \int_{0}^{\infty} \int_{K} u^{q} \varphi d x d t+\int_{K} u(x, 0) \varphi(x, 0) d x
\end{aligned}
$$

Введем параметры

$$
\begin{gathered}
s_{\alpha}^{*}=\frac{\alpha+N-2}{2}+\sqrt{\left(\frac{\alpha+N-2}{2}\right)^{2}+\lambda_{\omega}}, \\
s_{* \alpha}=-\frac{\alpha+N-2}{2}+\sqrt{\left(\frac{\alpha+N-2}{2}\right)^{2}+\lambda_{\omega}} .
\end{gathered}
$$

TEOPEMA 2. $\Pi p u$

$$
1<q \leqslant q^{*}=1+\frac{2-\alpha}{s_{\alpha}^{*}+2-\alpha}
$$

задача (4.1) не имеет нетривиального глобального решения.

ДокАЗАТЕльство. Пусть $u$ - нетривиальное слабое решение задачи (4.1). Выберем пробную функцию в виде, аналогичном модельной задаче, однако в качестве функции $\xi$ вместо (2.4) возьмем

$$
\xi_{\alpha}(x) \equiv \xi_{\alpha}(r, \omega)=r^{s_{* \alpha}} \Phi(\omega)
$$

Тогда функции $\psi_{\rho}(x)$ и $\varphi_{\rho}(x, t)$ принимают вид

$$
\psi_{\rho}(x)=\xi_{\alpha}(x) \eta\left(\frac{|x|}{\rho}\right), \quad \varphi_{\rho}(x, t)=\psi_{\rho}(x) \eta\left(\frac{t}{\rho^{\theta}}\right)
$$


В данном случае вместо оператора Лапласа мы имеем оператор

$$
A_{\alpha} \equiv \operatorname{div}\left(|x|^{\alpha} D\right)=r^{\alpha}\left(\frac{\partial^{2}}{\partial r^{2}}+\frac{\alpha+N-1}{r} \frac{\partial}{\partial r}+\frac{1}{r^{2}} \Delta_{\omega}\right) .
$$

Непосредственной подстановкой легко убедиться, что

$A_{\alpha} \xi_{\alpha} \equiv \operatorname{div}\left(|x|^{\alpha} D \xi_{\alpha}\right)=r^{\alpha} r^{s_{* \alpha}-2}\left(s_{* \alpha}\left(s_{* \alpha}-1\right)+s_{* \alpha}(\alpha+N-1)-\lambda_{\omega}\right) \Phi(\omega) \equiv 0$ в $K$.

Действуя как в $\S 2$, можно получить аналоги оценок (2.7):

$$
\begin{aligned}
& \iint_{\operatorname{supp}\left|\frac{\partial \varphi_{\rho}}{\partial t}\right|} \frac{\left|\frac{\partial \varphi_{\rho}(x, t)}{\partial t}\right|^{p}}{\varphi_{\rho}^{p-1}(x, t)} d x d t \\
& \quad \leqslant \int_{K \cap\{r<2 \rho\}} \psi_{\rho}(x) d x \int_{\operatorname{supp}\left|\frac{d \eta\left(t / \rho^{\theta}\right)}{d t}\right|} \frac{\left|\frac{d \eta\left(t / \rho^{\theta}\right)}{d t}\right|^{p}}{\eta^{p-1}\left(t / \rho^{\theta}\right)} d t \\
& \quad \leqslant c \rho^{s_{* \alpha}+N-\theta(p-1)}, \\
& \iint_{\operatorname{supp}\left|A_{\alpha} \varphi_{\rho}\right|} \frac{\left|A_{\alpha} \varphi_{\rho}(x, t)\right|^{p}}{\varphi^{p-1}(x, t)} d x d t \\
& \quad \leqslant \int_{0}^{2 \rho^{\theta}} \eta\left(\frac{t}{\rho^{\theta}}\right) d t \int_{\operatorname{supp}\left|A_{\alpha} \psi_{\rho}\right|} \frac{\left|A_{\alpha} \psi_{\rho}\right|^{p}}{\psi_{\rho}^{p-1}} d x \\
& \quad \leqslant c \rho^{\theta-(2-\alpha) p+s_{* \alpha}+N} .
\end{aligned}
$$

При $\theta=2-\alpha>0$ степени в правых частях оценок совпадают и тогда

$$
\iint_{\operatorname{supp}\left|\frac{\partial \varphi_{\rho}}{\partial t}+A_{\alpha} \varphi_{\rho}\right|} \frac{\left|\frac{\partial \varphi_{\rho}}{\partial t}+A_{\alpha} \varphi_{\rho}\right|^{p}}{\varphi_{\rho}^{p-1}} d x d t \leqslant c_{0} \rho^{-(2-\alpha) p+s_{* \alpha}+N+2-\alpha} .
$$

Далее действуем по схеме доказательства теоремы 1. Из определения слабого решения

$$
\begin{aligned}
& \int_{K} u(x, 0) \psi_{\rho}(x) d x+\int_{0}^{\infty} \int_{K} u^{q} \varphi_{\rho} d x d t \\
& \quad \leqslant \int_{0}^{\infty} \int_{\partial K} u|x|^{\alpha} \frac{\partial \varphi_{\rho}}{\partial n} d x d t+\iint_{\operatorname{supp}\left|\frac{\partial \varphi_{\rho}}{\partial t}+A_{\alpha} \varphi_{\rho}\right|} u\left|\frac{\partial \varphi_{\rho}}{\partial t}+A_{\alpha} \varphi_{\rho}\right| d x d t,
\end{aligned}
$$

откуда (с учетом неположительности первого интеграла в правой части) после применения неравенства Гёльдера получаем

$$
\begin{aligned}
\iint_{\varphi_{\rho}(x, t)=\xi_{\alpha}(x)} u^{q} \xi_{\alpha} d x d t & \leqslant \iint_{\operatorname{supp}\left|\frac{\partial \varphi_{\rho}}{\partial t}+A_{\alpha} \varphi_{\rho}\right|} \frac{\left|\frac{\partial \varphi_{\rho}}{\partial t}+A_{\alpha} \varphi_{\rho}\right|^{q^{\prime}}}{\varphi_{\rho}^{q^{\prime}-1}} d x d t \\
& \leqslant c_{0} \rho^{-(2-\alpha) q^{\prime}+s_{* \alpha}+N+2-\alpha}
\end{aligned}
$$

что дает условие отсутствия нетривиального решения

$$
-(2-\alpha) q^{\prime}+s_{* \alpha}+N+2-\alpha \leqslant 0 .
$$
Отсюда $q \leqslant 1+\frac{2-\alpha}{N+s_{* \alpha}}$. Так как $N+s_{* \alpha}=s_{\alpha}^{*}+2-\alpha$, то приходим к утверждению
теоремы. 
ЗАмЕчАниЕ 7. Существенно более общие классы параболических неравенств для случая всего пространства $\mathbb{R}^{N}$ рассмотрены, например, в работах [15] и [16]. Схожий с использованным способ выбора пробной функции в виде фундаментального решения эллиптического оператора (для всего пространства $\mathbb{R}^{N}$ ) использован в [17].

ЗАМЕчАниЕ 8 . Мы не рассмотрели случай $\alpha \geqslant 2$, ког да на отсутствие решения начинает оказывать влияние вырождение оператора при $x=0$. Данное явление получило название “полного разрушения решения". Одновременно можно ставить вопрос о времени существования решения и на этом пути получать утверждения о "мгновенном разрушении", т.е. об отсутствии даже локальных решений рассматриваемых задач [16], [18].

\section{§5. Зависимость критического показателя от начальных данных}

В предыдущем параграфе в процессе доказательства теоремы 2 при получении неравенства (4.4) была установлена по сути априорная оценка роста возможного решения задачи. Сформулируем ее в виде отдельного утверждения.

Лемма 1. Пусть и $(x, t)$ - решение задачи (4.1). Тогда при достаточно больиих $\rho$ и при любом $q>1$ справедлива оценка

$$
\begin{gathered}
\int_{K \cap\{|x|<\rho\}} u(x, 0) \xi_{\alpha}(x) d x+\iint_{(K \times(0, \infty)) \cap\{|x|<\rho\} \cap\left\{t<\rho^{2-\alpha}\right\}} u^{q} \xi_{\alpha} d x d t \\
\leqslant c_{1} \rho^{-(2-\alpha) q^{\prime}+s_{* \alpha}+N+2-\alpha}
\end{gathered}
$$

ДокАЗАТЕльство. Действуем аналогично доказательству теоремы 2, однако при переходе от формулы (4.4) к (4.5) учтем наличие в левой части интеграла от начального значения $u(x, 0)$ и приведем явное выражение для множества $\{(x, t)$ : $\left.\varphi_{\rho}(x, t)=\xi_{\alpha}(x)\right\}$.

Эта лемма позволяет исследовать вопрос о разрушении локального решения в зависимости от скорости роста при $|x| \rightarrow \infty$ значений функции $u(x, 0)$ (что соответствует в случае уравнения начальным данным). Приведем некоторые результаты в этом направлении.

ТЕОРема 3. Пусть $u(x, 0) \geqslant c_{\gamma} /|x|^{\gamma}$ при достаточно больиих $|x|$, где $\gamma>0$, $c_{\gamma}$ - постоянная. Тогда при

$$
1-N<\alpha<2, \quad 1<q<q_{c}(\gamma), \text { zдe } q_{c}(\gamma)=\max \left\{1+\frac{2-\alpha}{\gamma}, 1+\frac{2-\alpha}{s_{\alpha}^{*}+2-\alpha}\right\}
$$

задача (4.1) не имеет глобального нетривиального решения.

ДоказАТЕльство. Пусть $\gamma \geqslant s_{\alpha}^{*}+2-\alpha=s_{* \alpha}+N$. Тогда $q_{c}(\gamma)=1+$ $\frac{2-\alpha}{s_{\alpha}^{*}+2-\alpha}$ и задача (4.1) не имеет глобального нетривиального решения по теореме 2. Поэтому рассмотрим оставшийся вариант: $0<\gamma<s_{* \alpha}+N$, когда $q_{c}=1+(2-\alpha) / \gamma$. 
Пусть $u$ - глобальное решение, удовлетворяюшее условиям теоремы. Для определенности считаем, что $u(x, 0) \geqslant c_{\gamma} /|x|^{\gamma}$ при $|x|>R$. Применяя лемму 1 , получаем при $\rho>2 R$

$$
\begin{aligned}
c_{1} \rho^{-(2-\alpha) q^{\prime}+s_{* \alpha}+N+2-\alpha} \geqslant & \int_{K \cap\{\rho / 2<|x|<\rho\}} u(x, 0) \xi_{\alpha} d x \\
& +\iint_{(K \times(0, \infty)) \cap\{|x|<\rho\} \cap\left\{t<\rho^{2-\alpha}\right\}} u^{q} \xi_{\alpha} d x d t \\
\geqslant & c_{\gamma} \int_{\rho / 2}^{\rho} r^{N-1} \frac{r^{s_{* \alpha}}}{r^{\gamma}} d r \int_{K_{\omega}} \Phi(\omega) d \omega \geqslant c_{\gamma} c_{2} \rho^{s_{* \alpha}-\gamma+N} .
\end{aligned}
$$

Из приведенной оценки следует неравенство

$$
c_{\gamma} c_{2} \rho^{(2-\alpha)\left(q^{\prime}-1\right)-\gamma} \leqslant c_{1}
$$

которое в условиях теоремы не может удовлетворяться при $\rho \rightarrow \infty$, так как если $q<1+(2-\alpha) / \gamma$, то $(2-\alpha)\left(q^{\prime}-1\right)-\gamma>0$.

Случай $\gamma=0$ выделим отдельно.

Теорема 4. Пусть $u(x, 0) \geqslant c>0$ для $|x| \rightarrow \infty$. Тогда задача (4.1) не имеет решения для всех $q>1$.

ДокАЗАТЕЛьство. Применяя лемму 1, вместо (5.1) получаем неравенство $c c_{2} \rho^{(2-\alpha)\left(q^{\prime}-1\right)} \leqslant c_{1}$, которое не может выполняться в условиях теоремы, так как $\alpha<2$ и $q^{\prime}>1$.

Теорему 3 с использованием оценки (5.1) можно сформулировать также и в виде условия на рост начальных данных.

Теорема 5. Пусть $u(x, 0) \geqslant c_{\gamma} /|x|^{\gamma}$ при достаточно больиих $|x|$. Тогда $n p u$

$$
1-N<\alpha<2, \quad q>1, \quad 0<\gamma<\frac{2-\alpha}{q-1}
$$

задача (4.1) не имеет глобального нетривиального решения.

ЗАмЕчАнИЕ 9. В работе [21] для задачи $u_{t}-\Delta u=|x|^{\sigma} u^{q}$ установлено, что в предельном случае $\gamma=\frac{2+\sigma}{q-1}$ задача также не имеет нетривиального решения. В нашем доказательстве при достаточно больших значениях $c_{\gamma}$ отсутствие решения в предельном случае очевидно.

ЗАмЕчАниЕ 10 . Используемьй способ в случае задач в $\mathbb{R}^{N+1}$ изложен в [16].

\section{§6. Неоднородная задача}

Следуя статье [22], введем в систему (4.1) дополнительное слагаемое $w(x) \geqslant 0$, $w(x) \in L_{1, \text { loc }}(K)$, т.е. рассмотрим задачу:

$$
\begin{gathered}
\frac{\partial u}{\partial t}-\operatorname{div}\left(|x|^{\alpha} D u\right) \geqslant u^{q}+w(x) \quad \text { в } K \times(0, \infty), \quad w(x) \not \equiv 0, \\
u \geqslant 0, \quad u \neq 0 .
\end{gathered}
$$


Здесь, как и ранее, $2>\alpha>1-N$.

Нас интересует вопрос о том, как повлияет введение неоднородности на критический показатель отсутствия решения.

Слабое решение этой задачи понимается в смысле определения 2, т.е. предполагается выполненным интегральное неравенство

$$
\begin{aligned}
\int_{0}^{\infty} & \int_{\partial K} u|x|^{\alpha} \frac{\partial \varphi}{\partial n} d x d t-\int_{0}^{\infty} \int_{K} u\left(\frac{\partial \varphi}{\partial t}+\operatorname{div}\left(|x|^{\alpha} D \varphi\right)\right) d x d t \\
& \geqslant \int_{0}^{\infty} \int_{K} u^{q} \varphi d x d t+\int_{0}^{\infty} \int_{K} w(x) \varphi d x d t+\int_{K} u(x, 0) \varphi(x, 0) d x
\end{aligned}
$$

TeOpema 6. При

$$
1<q<q^{*}=1+\frac{2-\alpha}{s_{\alpha}^{*}}
$$

задача (6.1) не имеет нетривиального глобального решения, каким бы мальм ни бълло $w(x) \not \equiv 0$.

ДокАЗАТЕЛьство. Следуя доказательству теоремы 2 и леммы 1 , получаем оценку

$$
\begin{gathered}
\int_{0}^{\rho^{2-\alpha}} \int_{K \cap\{|x|<\rho\}} w(x) \xi_{\alpha}(x) d x d t+\int_{K \cap\{|x|<\rho\}} u(x, 0) \xi_{\alpha}(x) d x \\
\quad+\iint_{\varphi_{\rho}(x, t)=\xi \alpha(x)} u^{q_{\xi_{\alpha}} d x d t \leqslant c_{1} \rho^{-(2-\alpha) q^{\prime}+s_{* \alpha}+N+2-\alpha}}
\end{gathered}
$$

откуда

$$
c_{1} \rho^{-(2-\alpha) q^{\prime}+s_{* \alpha}+N+2-\alpha} \geqslant \int_{0}^{\rho^{2-\alpha}} \int_{K \cap\{|x|<\rho\}} w(x) \xi_{\alpha}(x) d x d t \geqslant \rho^{2-\alpha} c_{w}
$$

при $\rho$ таких, что

$$
\int_{K \cap\{|x|<\rho\}} w(x) \xi_{\alpha}(x) d x \geqslant c_{w} \equiv \text { const }>0 .
$$

Для получения противоречия при $\rho \rightarrow \infty$ достаточно предполагать, что

$$
-(2-\alpha) q^{\prime}+s_{* \alpha}+N<0
$$

т.e.

$$
q<1+\frac{2-\alpha}{s_{\alpha}^{*}}
$$

Теорема доказана.

ЗАмЕчАниЕ 11. Сравнение с теоремой 2 показывает, что при введении в неравенство возмущения $w(x) \geqslant 0$ критический показатель изменяется в сторону увеличения. Интересным моментом в данном утверждении является отсутствие предположений о росте и носителе функции $w(x)$, требуется только положительность интеграла по некоторой достаточно большой сфере в $\mathbb{R}^{N}$. Объяснение этому кроется в интегрировании функции $w(x)$ по переменной $t$ в пределах от 0 до $\rho^{2-\alpha}$, за счет чего для любой сколь угодно малой функции $w(x)$ все равно получается стремящийся к бесконечности интеграл (при $\rho \rightarrow \infty)$. 
Теорема 7. Пусть $w(x) \geqslant c_{\gamma} /|x|^{\gamma}$, где $\gamma>2-\alpha>0$. Тогда задача (6.1) не имеет глобального нетривиального решения при $1-N<\alpha<2$ и $1<q<$ $\gamma /(\gamma+\alpha-2)$.

ДокАЗАТЕЛЬСтво. В условиях теоремы неравенство (6.2) принимает вид

$$
c_{1} \rho^{-(2-\alpha) q^{\prime}+s_{* \alpha}+N+2-\alpha} \geqslant \rho^{2-\alpha} \rho^{s_{* \alpha}+N-\gamma} c_{w}
$$

откуда $c_{1} \geqslant c_{w} \rho^{(2-\alpha) q^{\prime}-\gamma}$. Неравенство невозможно, если $(2-\alpha) q^{\prime}-\gamma>0$, т.е. $q<\gamma /(\gamma+\alpha-2)$.

Можно рассмотреть неоднородную задачу с возмушением $w(t) \geqslant 0$, зависящим только от переменной $t$ :

$$
\begin{gathered}
\frac{\partial u}{\partial t}-\operatorname{div}\left(|x|^{\alpha} D u\right) \geqslant u^{q}+w(t) \quad \text { в } K \times(0, \infty), \\
u \geqslant 0, \quad u \neq 0 .
\end{gathered}
$$

Теорема 8. При всех $q>1 u 2>\alpha>1-N$ задача (6.3) не имеет нетривиального глобального решения, каким бъ малым ни было $w(t) \not \equiv 0$.

ДокАЗАТЕЛЬство. Аналогично доказательству теоремы 6 получим оценку

$$
c_{1} \rho^{-(2-\alpha) q^{\prime}+s_{* \alpha}+N+2-\alpha} \geqslant \int_{K \cap\{|x|<\rho\}} \xi_{\alpha}(x) d x \int_{0}^{\rho^{2-\alpha}} w(t) d t \geqslant \rho^{s_{* \alpha}+N} c_{w}
$$

для $\rho$ таких, что

$$
\int_{0}^{\rho^{2-\alpha}} w(t) d t \geqslant c_{w} \equiv \text { const }>0
$$

В условиях теоремы справедливо неравенство $-(2-\alpha)\left(q^{\prime}-1\right)<0$, которое показывает, что соотношение (6.4) не может выполняться, следовательно, задача (6.3) не имеет нетривиального решения.

\section{§7. Квазилинейное неравенство типа медленной диффузии}

В последнее время большой интерес вызывают дифференциальные неравенства вида

$$
\begin{gathered}
\frac{\partial u}{\partial t}-\Delta u^{m} \geqslant u^{q} \text { в } K \times(0, \infty), \quad m>1, \quad q>m, \\
u \geqslant 0, \quad u \neq 0 .
\end{gathered}
$$

При внешне нелинейной структуре к их исследованию часто можно применить методы, разработанные для полулинейных задач.

Далее слабое решение будет пониматься в следуюшем смысле. 
ОПРЕДЕЛЕНИЕ 3 . Пусть $u(x, t) \in C(\bar{K} \times[0, \infty))$. Неотрицательная функция $u(x, t)$ называется слабым решением задачи (7.1), если для любой неотрицательной пробной функции $\varphi(x, t) \in W_{\infty}^{2,1}(K \times(0, \infty))$ такой, что $\left.\varphi\right|_{\partial K \times(0, \infty)}=0$, финитной по переменным $r=|x|$ и $t$, выполнено интегральное неравенство

$$
\begin{aligned}
\int_{0}^{\infty} & \int_{\partial K} u \frac{\partial \varphi}{\partial n} d x d t-\int_{0}^{\infty} \int_{K} u \frac{\partial \varphi}{\partial t} d x d t-\int_{0}^{\infty} \int_{K} u^{m} \Delta \varphi d x d t \\
& \geqslant \int_{0}^{\infty} \int_{K} u^{q} \varphi d x d t+\int_{K} u(x, 0) \varphi(x, 0) d x
\end{aligned}
$$

TЕOРЕMA 9. $\Pi p u$

$$
1<m<q \leqslant q^{*}=m+\frac{2}{s^{*}+2}
$$

задача (7.1) не имеет нетривиального глобального решения. Число $s^{*}$ определено в (2.3).

ДокАЗАТЕЛьство аналогично доказательству теоремы 1. Выбор пробной функции естественным образом обобщает предыдушее построение: по переменной $t$ вводится некоторый вес $\theta>0$, который уточним позднее (аналогично работе [17]), т.е. берем пробную функцию (2.6).

Пусть $u(x, t)$ - нетривиальное решение задачи (7.1) с $1<q \leqslant q^{*}$. Рассмотрим неравенство (7.2) из определения 3 обобшенного решения с пробной функцией $\varphi_{\rho}$. Первый интеграл в левой части (по границе $\partial K$ ) неположителен в силу приведенных в доказательстве теоремы 1 аргументов. Применяя неравенство Гёльдера ко второму и третьему интегралам из левой части (7.2), получаем

$$
\begin{aligned}
& \int_{K} u(x, 0) \psi_{\rho}(x) d x+\int_{0}^{\infty} \int_{K} u^{q} \varphi_{\rho} d x d t \\
& \quad \leqslant\left(\iint_{\operatorname{supp}\left|\frac{\partial \varphi_{\rho}}{\partial t}\right|} u^{q} \varphi_{\rho} d x d t\right)^{1 / q}\left(\iint_{\operatorname{supp}\left|\frac{\partial \varphi_{\rho}}{\partial t}\right|} \frac{\left|\frac{\partial \varphi_{\rho}}{\partial t}\right|^{q^{\prime}}}{\varphi_{\rho}^{q^{\prime}-1}} d x d t\right)^{1 / q^{\prime}} \\
& \quad+\left(\iint_{\operatorname{supp}\left|\Delta \varphi_{\rho}\right|} u^{q} \varphi_{\rho} d x d t\right)^{m / q}\left(\iint_{\operatorname{supp}\left|\Delta \varphi_{\rho}\right|} \frac{\left|\Delta \varphi_{\rho}\right|^{q /(q-m)}}{\varphi_{\rho}^{m /(q-m)}} d x d t\right)^{(q-m) / q}
\end{aligned}
$$

Оценки (2.7) в этом случае (заметим, что в первом неравенстве из (2.7) надо брать $p=q^{\prime}$, тогда как во втором $\left.p=q /(q-m)\right)$ принимают вид

$$
\begin{gathered}
\iint_{\operatorname{supp}\left|\frac{\partial \varphi_{\rho(x, t)}}{\partial t}\right|} \frac{\left|\frac{\partial \varphi_{\rho}(x, t)}{\partial t}\right|^{q^{\prime}}}{\varphi_{\rho}^{q^{\prime}-1}} d x d t \leqslant c \rho^{-\theta q^{\prime}+s_{*}+\theta+N}, \\
\iint_{\operatorname{supp}\left|\Delta \varphi_{\rho}(x, t)\right|} \frac{\left|\Delta \varphi_{\rho}\right|^{q /(q-m)}}{\varphi_{\rho}^{m /(q-m)}} d x d t \leqslant c \rho^{-2 q /(q-m)+s_{*}+\theta+N} .
\end{gathered}
$$

Теперь подбираем параметр $\theta$ таким образом, чтобы показатели сравнялись [17]:

$$
-\theta q^{\prime}+s_{*}+\theta+N=-\frac{2 q}{q-m}+s_{*}+\theta+N, \quad \text { т.е. } \theta=\frac{2(q-1)}{q-m} .
$$


Заметим, что $\theta>0$. При таком выборе $\theta$ из неравенства (7.3) будем иметь

$$
\iint_{\varphi_{\rho}(x, t)=\xi(x)} u^{q} \xi d x d t \leqslant c_{0} \rho^{-2 /(q-m)+s_{*}+N}
$$

В случае

$$
-\frac{2}{q-m}+s_{*}+N \leqslant 0
$$

с использованием аналогичных предыдущим рассуждений это означает отсутствие нетривиального решения при $1<m<q \leqslant q^{*}=m+\frac{2}{s_{*}+N}=m+\frac{2}{s^{*}+2}$.

ЗАмечАниЕ 12 . Для всего пространства $\mathbb{R}^{N}$ (т.е. $s^{*}=N-2$ ) получаем известный результат об отсутствии нетривиального решения при $m<q \leqslant m+2 / N$.

ЗАмЕчАниЕ 13. Сделав замену $u^{m}=v$, от (7.1) приходим к задаче

$$
\frac{\partial v^{1 / m}}{\partial t}-\Delta v \geqslant v^{q / m}
$$

для которой легко переформулировать полученное выше утверждение.

Можно обобщить представленную теорему на неравенства с переменными коэффициентами вида

$$
\frac{\partial u}{\partial t}-\operatorname{div}\left(|x|^{\alpha} D\left(u^{m}\right)\right) \geqslant u^{q},
$$

а также установить зависимость критического показателя от поведения начальных данных (методами $§ 5$ ) и изменение критического показателя при введении в задачу неоднородности (методами $\S 6$ ).

\section{§ 8. Системы неравенств}

Рассмотрим систему

$$
\left\{\begin{array}{l}
\frac{\partial u}{\partial t}-\operatorname{div}\left(|x|^{\alpha} D u\right) \geqslant v^{q_{1}} \quad \text { в } K \times(0, \infty) \\
\frac{\partial v}{\partial t}-\operatorname{div}\left(|x|^{\alpha} D v\right) \geqslant u^{q_{2}} \quad \text { в } K \times(0, \infty) \\
u \geqslant 0, \quad v \geqslant 0, \quad u \neq 0, \quad v \not \equiv 0
\end{array}\right.
$$

где $2>\alpha>1-N$. Такого рода системы часто называют слабо связанными.

ОПРЕДЕЛЕНИЕ 4. Пара неотрицательных функций $u, v \in C(\bar{K} \times[0, \infty))$ называется слабым решением задачи (8.1), если для любой неотрицательной пробной 
функции $\varphi(x, t) \in W_{\infty}^{2,1}(K \times(0, \infty))$ такой, что $\left.\varphi\right|_{\partial K \times(0, \infty)}=0$, финитной по переменньм $r=|x|$ и $t$, выполнены интегральные неравенства

$$
\begin{aligned}
\int_{0}^{\infty} & \int_{\partial K} u|x|^{\alpha} \frac{\partial \varphi}{\partial n} d x d t-\int_{0}^{\infty} \int_{K} u\left(\frac{\partial \varphi}{\partial t}+\operatorname{div}\left(|x|^{\alpha} D \varphi\right)\right) d x d t \\
& \geqslant \int_{0}^{\infty} \int_{K} v^{q_{1}} \varphi d x d t+\int_{K} u(x, 0) \varphi(x, 0) d x, \\
\int_{0}^{\infty} & \int_{\partial K} v|x|^{\alpha} \frac{\partial \varphi}{\partial n} d x d t-\int_{0}^{\infty} \int_{K} v\left(\frac{\partial \varphi}{\partial t}+\operatorname{div}\left(|x|^{\alpha} D \varphi\right)\right) d x d t \\
& \geqslant \int_{0}^{\infty} \int_{K} u^{q_{2}} \varphi d x d t+\int_{K} v(x, 0) \varphi(x, 0) d x .
\end{aligned}
$$

ТЕОРема 10. Задача (8.1) не имеет слабого решения, если

$$
\max \left\{\gamma_{1}, \gamma_{2}\right\} \geqslant \frac{s_{\alpha}^{*}+2-\alpha}{2-\alpha},
$$

əде

$$
\gamma_{1}=\frac{q_{1}+1}{q_{1} q_{2}-1}, \quad \gamma_{2}=\frac{q_{2}+1}{q_{1} q_{2}-1},
$$

$s_{\alpha}^{*}$ определяется формулой (4.2).

ДокАЗАТЕЛьство. Пусть $u, v$ - слабое решение задачи (8.1). Выберем пробную функцию как в доказательстве теоремы 2 и будем использовать соответствующие обозначения. В частности, справедлива оценка (4.3).

Применяя в определении слабого решения неравенство Гёльдера, приходим к неравенствам

$$
\begin{aligned}
& \int_{K} u(x, 0) \varphi_{\rho}(x, 0) d x+\int_{0}^{\infty} \int_{K} v^{q_{1}} \varphi_{\rho} d x d t \\
& \leqslant\left(\iint_{\operatorname{supp}\left|\frac{\partial \varphi_{\rho}}{\partial t}+A_{\alpha} \varphi_{\rho}\right|} u^{q_{2}} \varphi_{\rho} d x d t\right)^{1 / q_{2}} \\
& \times\left(\iint_{\operatorname{supp}\left|\frac{\partial \varphi_{\rho}}{\partial t}+A_{\alpha} \varphi_{\rho}\right|} \frac{\left|\frac{\partial \varphi_{\rho}}{\partial t}+A_{\alpha} \varphi_{\rho}\right|^{q_{2}^{\prime}}}{\varphi_{\rho}^{q_{2}^{\prime}-1}} d x d t\right)^{1 / q_{2}^{\prime}} \\
& \equiv\left(\iint_{\operatorname{supp}\left|\frac{\partial \varphi_{\rho}}{\partial t}+A_{\alpha} \varphi_{\rho}\right|} u^{q_{2}} \varphi_{\rho} d x d t\right)^{1 / q_{2}} J_{1}^{1 / q_{2}^{\prime}}, \\
& \int_{K} v(x, 0) \varphi_{\rho}(x, 0) d x+\int_{0}^{\infty} \int_{K} u^{q_{2}} \varphi_{\rho} d x d t \\
& \leqslant\left(\iint_{\operatorname{supp}\left|\frac{\partial \varphi_{\rho}}{\partial t}+A_{\alpha} \varphi_{\rho}\right|} v^{q_{1}} \varphi_{\rho} d x d t\right)^{1 / q_{1}} \\
& \times\left(\iint_{\operatorname{supp}\left|\frac{\partial \varphi_{\rho}}{\partial t}+A_{\alpha} \varphi_{\rho}\right|} \frac{\left|\frac{\partial \varphi_{\rho}}{\partial t}+A_{\alpha} \varphi_{\rho}\right|^{q_{1}^{\prime}}}{\varphi_{\rho}^{q_{1}^{\prime}-1}} d x d t\right)^{1 / q_{1}^{\prime}} \\
& \equiv\left(\iint_{\operatorname{supp}\left|\frac{\partial \varphi_{\rho}}{\partial t}+A_{\alpha} \varphi_{\rho}\right|} v^{q_{1}} \varphi_{\rho} d x d t\right)^{1 / q_{1}} J_{2}^{1 / q_{1}^{\prime}}
\end{aligned}
$$


причем согласно (4.3)

$$
J_{1} \leqslant c_{0} \rho^{-(2-\alpha) q_{2}^{\prime}+s_{* \alpha}+N+2-\alpha}, \quad J_{2} \leqslant c_{0} \rho^{-(2-\alpha) q_{1}^{\prime}+s_{* \alpha}+N+2-\alpha} .
$$

Подставим неравенство (8.3) в (8.2). Тогда

$$
\begin{aligned}
& \int_{K} u(x, 0) \psi_{\rho}(x) d x+\int_{0}^{\infty} \int_{K} v^{q_{1}} \varphi_{\rho} d x d t \\
& \quad \leqslant\left(\iint_{\operatorname{supp}\left|\frac{\partial \varphi_{\rho}}{\partial t}+A_{\alpha} \varphi_{\rho}\right|} v^{q_{1}} \varphi_{\rho} d x d t\right)^{1 /\left(q_{1} q_{2}\right)} J_{2}^{1 /\left(q_{1}^{\prime} q_{2}\right)} J_{1}^{1 / q_{2}^{\prime}}
\end{aligned}
$$

откуда после упрошения степеней получаем

$$
\int_{0}^{\infty} \int_{K} v^{q_{1}} \varphi_{\rho} d x d t \leqslant\left(J_{2}^{q_{1}-1} J_{1}^{q_{1}\left(q_{2}-1\right)}\right)^{1 /\left(q_{1} q_{2}-1\right)} \leqslant C \rho_{\alpha}^{s^{*}+2-\alpha-\gamma_{1}(2-\alpha)} .
$$

Отсюда, рассуждая как и раньше, выводим отсутствие нетривиального $v(x, t)$ в случае

$$
s_{\alpha}^{*}+2-\alpha-\gamma_{1}(2-\alpha) \leqslant 0 .
$$

Аналогично, подставляя (8.2) в (8.3), приходим к оценке

$$
\int_{0}^{\infty} \int_{K} u^{q_{2}} \varphi_{\rho} d x d t \leqslant\left(J_{1}^{q_{2}-1} J_{2}^{q_{2}\left(q_{1}-1\right)}\right)^{1 /\left(q_{1} q_{2}-1\right)} \leqslant C \rho_{\alpha}^{s_{\alpha}^{*}+2-\alpha-\gamma_{2}(2-\alpha)}
$$

т.е. нетривиальное решение $u(x, t)$ отсутствует при

$$
s_{\alpha}^{*}+2-\alpha-\gamma_{2}(2-\alpha) \leqslant 0 .
$$

Очевидно, что если хотя бы одна из функций $u(x, t)$ или $v(x, t)$ тождественно равна нулю, то равна нулю и другая. Таким образом, условие отсутствия нетривиального решения принимает вид

$$
\max \left\{\gamma_{1}, \gamma_{2}\right\} \geqslant \frac{s_{\alpha}^{*}+2-\alpha}{2-\alpha}
$$

Теорема доказана.

При $\alpha=0$ получаем известные условия

$$
\max \left\{\frac{q_{1}+1}{q_{1} q_{2}-1}, \frac{q_{2}+1}{q_{1} q_{2}-1}\right\} \geqslant \frac{s^{*}+2}{2},
$$

которые для всего пространства $\mathbb{R}^{N}$ принимают вид (полагаем $s^{*}=N-2$ )

$$
\max \left\{\frac{q_{1}+1}{q_{1} q_{2}-1}, \frac{q_{2}+1}{q_{1} q_{2}-1}\right\} \geqslant \frac{N}{2} .
$$

ЗАмЕчАнИЕ 14. Возможно применить предлагаемый метод к более общим системам. В частности, не вызывает проблем изучение систем с постоянными положительными коэффициентами вида

$$
\left\{\begin{array}{l}
a_{11} \frac{\partial u}{\partial t}-a_{12} \operatorname{div}\left(|x|^{\alpha} D u\right) \geqslant v^{q_{1}}, \\
a_{21} \frac{\partial v}{\partial t}-a_{22} \operatorname{div}\left(|x|^{\alpha} D v\right) \geqslant u^{q_{2}}
\end{array} \text { в } K \times(0, \infty) .\right.
$$

Результат теоремы 10 остается верен и для этой системы (с ненулевыми коэффициентами).

В заключение автор выражает благодарность С.И. Похожаеву за постановку задачи и В.В. Курте за полезное обсуждение результатов. 


\section{Список литературы}

1. Galaktionov V. A., Vazquez J. L. The problem of blow-up in nonlinear parabolic equations // Preprint. Univ. Bath. (UK), 2000. Math. 00/04.

2. Кондратьев B. A. Краевые задачи для эллиптических уравнений в областях с коническими и угловьми точками // Труды ММО. 1967. Т. 16. С. 209-292.

3. Bandle C., Levine H.A. On the existence and nonexistence of global solutions of reaction-diffusion equations in sectorial domains // Trans. Amer. Math. Soc. 1989. V. 316. P. 595-622.

4. Levine H. A., Meier P. The value of the critical exponent for reaction-diffusion equations in cones // Arch. Rational Mech. Anal. 1990. V. 109. P. 73-80.

5. Самарский А.А., Галактионов В.А., Курдюмов С. П., Михайлов А. П. Режимыс обострением в задачах для квазилинейных параболических уравнений. М.: Наука, 1987.

6. Levine H. A. The role of critical exponents in blow-up theorems // SIAM Rev. 1990. V. 32. P. 371-386.

7. Deng K., Levine H.A. The role of critical exponent in blow-up theorems: the sequel // J. Math. Anal. Appl. 2000. V. 243. P. 85-126.

8. Коньков $A$. A. О неотрицательных решениях квазилинейных эллиптических неравенств // Изв. РАН. Сер. матем. 1999. Т. 63. № 2. С. 41-127.

9. Kon'kov A. A. Behavior of solutions of nonlinear second-order elliptic inequalities // Nonlinear Anal. 2000. V. 42. № 7. P. 1253-1270.

10. Кондратьев B. А., Ландис E. M. О качественнштх свойствах решений одного нелинейного уравнения второго порядка // Матем. сб. 1988. Т. 135. № 3. С. 346-360.

11. Нгуен Мань Хунг. Об отсутствии положительных решений нелинейных эллиптических уравнений второго порядка в конических областях // Дифференц. уравнения. 1998. Т. 34. № 4. C. 533-539.

12. Курта В. В. Об отсутствии положительных решений у полулинейных эллиптических уравнений // Труды МИАН. 1999. Т. 227. С. 162-169.

13. Курта В. В. Некоторые вопросы качественной теории нелинейных дифференциальных уравнений второго порядка // Дис. ... докт. физ.-матем. наук. М.: МИАН, 1994.

14. Митидиери Э., Похожсаев С. И. Отсутствие положительных решений для квазилинейных эллиптических задач в $\mathbb{R}^{N}$ // Труды МИАН. 1999. Т. 227. С. 192-222.

15. Pohozaev S., Tesei A. Blow-up of nonnegative solutions to quasilinear parabolic inequalities // Atti Accad. Naz. Lincei Cl. Sci. Fis. Mat. Natur. Rend. Lincei (9) Mat. Appl. 2000. V. 11. № 2. P. 99-109.

16. Митидиери Э., Похожаев С.И. Априорные оценки и отсутствие решений диффференциальных неравенств в частных производных // Труды МИАН. 2001. Т. 234 (в печати).

17. Mitidieri E., Pohozaev S. I. Nonexistence of weak solutions for some degenerate elliptic and parabolic problems on $\mathbb{R}^{n} / / \mathrm{J}$. Evolution Equat. (to appear).

18. Лаптев Г. Г. Об отсутствии решений одного класса сингулярных полулинейных дифференциальных неравенств // Труды МИАН. 2001. Т. 232. С. 223-235.

19. Лаптев Г. Г. Отсутствие глобальных положителњных решений систем полулинейных эллиптических неравенств в конусах // Изв. РАН. Сер. матем. 2000. Т. 64 . №6. C. $107-124$.

20. Бесов О. В., Ильин В. П., Никольский С. М. Интегральные представления функций и теоремы вложения. М.: Наука, 1996.

21. Hamada T. On the existence and nonexistence of global solutions of semilinear parabolic equations with slowly decaying initial data // Tsukuba J. Math. 1997. V. 21. P. 505-514.

22. Zhang $Q$. A new critical phenomenon for semilinear parabolic problems // J. Math. Anal. Appl. 1998. V. 219. P. 125-139.

Тульский государственньй университет

E-mail : laptev@home.tula.net 